\title{
Electron-phonon equilibration in laser-heated gold films
}

\author{
T. G. White,,${ }^{1,2, *}$ P. Mabey, ${ }^{2}$ D. O. Gericke, ${ }^{3}$ N. J. Hartley, ${ }^{2}$ H. W. Doyle, ${ }^{2}$ D. McGonegle, ${ }^{2}$ D. S. Rackstraw, ${ }^{2}$ \\ A. Higginbotham, ${ }^{2}$ and G. Gregori ${ }^{2}$ \\ ${ }^{1}$ Institute of Shock Physics, Imperial College London, London, United Kingdom \\ ${ }^{2}$ Department of Physics, University of Oxford, Parks Road, Oxford OX1 3PU, United Kingdom \\ ${ }^{3}$ Centre for Fusion, Space and Astrophysics, Department of Physics, University of Warwick, Coventry CV4 7AL, United Kingdom
}

(Received 22 April 2014; revised manuscript received 8 July 2014; published 23 July 2014)

\begin{abstract}
By irradiating a thin metal foil with an intense short-pulse laser, we have created a uniform system far from equilibrium. The deposited energy is initially deposited only within the electronic subsystem, and the subsequent evolution of the system is determined by the details of the electron-phonon coupling. Here, we measure the time evolution of the lattice parameter through multilayer Bragg diffraction and compare the result to classical molecular dynamic simulations to determine the lattice temperature. The electron-ion coupling constant for gold is then determined by comparison with the evolution of a two-temperature electron-phonon system.
\end{abstract}

DOI: 10.1103/PhysRevB.90.014305

PACS number(s): 52.38.-r, 46.40.-f, 02.70.-c, 71.38.-k

\section{INTRODUCTION}

The electron-phonon coupling within a material is an important property that determines or modifies many electronic properties such as electronic and thermal conductivities (with superconductivity as the premier example [1]), the magnetic susceptibility, the available electron energies, and thus, the equation of state [2]. Accordingly, this quantity is needed for a number of applications in nanotechnology [3], material science [4], laser ablation [5], and energy production by means of inertial confinement fusion [6].

A direct way of investigating electron-phonon coupling, or more generally electron-ion energy transfer, is to setup a nonequilibrium situation and observe the equilibration of the system towards a state with a common temperature. Excitation with short-pulse lasers is a well-established procedure to create such nonequilibrium systems. Since optical lasers directly interact only with the electrons in the sample, the electron subsystem contains a greater amount of energy after the heating process. After an ultrafast thermalization within the electron subsystem $[7,8]$, the energy then flows into the lattice by excitation of phonons, and ultimately ion-acoustic waves that heat the background ions [9]. This process is relatively slow, and electron and ions may have unequal temperatures for times that long exceed the laser pulse duration [10]. The evolution of the electron and phonon temperatures is thus a complex process which depends on many factors such as initial temperatures, crystal structure, defects, and scattering properties of the sample $[11,12]$.

Although energy relaxation between the electron and ion subsystems has been investigated in many studies both experimentally and theoretically, the process is not well understood. While experiments near equilibrium [9] show good agreement with standard theoretical predictions [11], other experiments with high excitation densities or a more complex material structure have demonstrated large deviations with relaxation times longer than predicted $[10,13]$. Such deviations are typical for strongly heated, fluid systems [14-18] where the energy transfer does not evolve through electron-phonon coupling but through direct two-particle scattering or ion acoustic

*thomas.white@imperial.ac.uk modes. Although theoretical models considering the collective behavior of these coupled system show similar effects [19-21], the predictions do not agree with the small amount of experimental data available.

Here, we investigate electron-phonon coupling in weakly driven gold samples, adding more data for non-equilibrium systems with low excitations. Indeed, our systems are heated only slightly above room temperature, requiring precise determination of the ion temperature. This is done through the determination of the response of the ion lattice via X-ray Bragg scattering. The time-dependent lattice constant obtained can then be related to the lattice temperature and, thus, the energy received from the electrons. This work differs from previous experiments, as in Ref. [9] where a similar experimental technique had been employed through utilization of largescale nonequilibrium molecular dynamics (MD) simulations to analyze the lattice response. These simulation contain up to $\sim 300000$ atoms and are able to model the full depth of the gold samples. It is this capability to fully capture the accoustic modes of the system which suggests that MD would be ideally suited to model systems with higher excitation densities.

\section{EXPERIMENT}

The experiment was performed using an ultrafast Ti:sapphire laser with wavelength centered at $800 \mathrm{~nm}, 50 \mathrm{fs}$ duration, and delivering $\sim 40 \mathrm{~mJ}$ per pulse. The laser was used in single-shot mode, and split into two equal arms to achieve a pump-probe geometry, as shown in Fig. 1. The error on the delay stage was determined from the precision of the micrometer stage to be less than 1 ps. One arm (the pump) was focused to a spot size of approximately $3 \mathrm{~mm}$ onto a $200 \mathrm{~nm}$ thick gold nanofoil [22]. This leads to a laser fluence incident on the sample of $0.28 \mathrm{~J} / \mathrm{cm}^{2}$. Taking into account the reflectivity of gold at $800 \mathrm{~nm}$, we estimate the energy flux on the target to be $\sim 0.01 \mathrm{~J} / \mathrm{cm}^{2}$. This corresponds to an overall increase in the energy of the sample on the order of $\sim 1 \mathrm{eV} / \mathrm{nm}^{3}$. The gold foil is grown on a mica substrate on the opposite side to the laser beam, whereas the laser side of the gold film only faces the vacuum and can expand freely.

The second arm (the probe) of the laser pulse was sent through a delay stage with difference in time of arrival of the two arms ranging from $\Delta t=-5 \mathrm{ps}$ to $\Delta t=250 \mathrm{ps}$ (where 


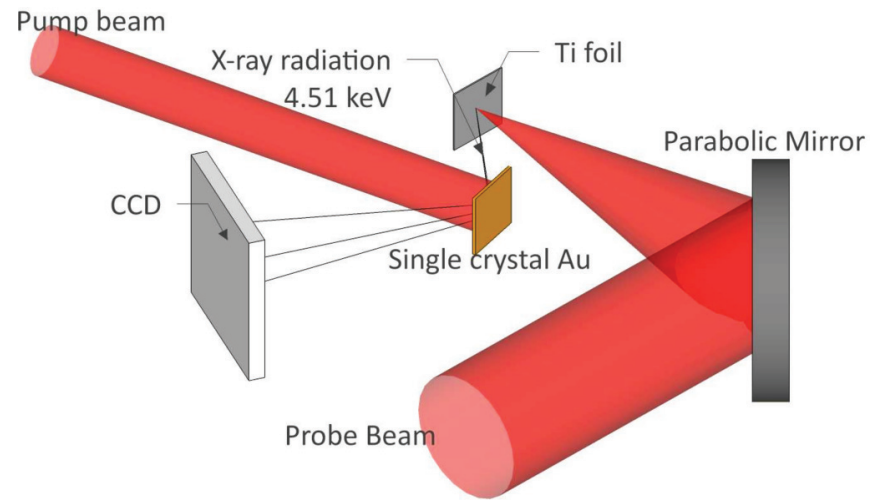

FIG. 1. (Color online) Schematic showing the experimental setup. The $50 \mathrm{fs}, 40 \mathrm{~mJ}, 800 \mathrm{~nm}$ laser is split into two arms. The first arm is focused onto the gold foil to rapidly heat the conduction electrons in the sample while the second arm passes through a delay stage and then drives the Ti $K-\alpha$ transition in a thin Ti foil in order to probe of the lattice parameter. The movement of the diffraction line due to the heating is imaged onto a CCD placed $10 \mathrm{~cm}$ away.

negative values means that the probe was fired earlier than the pump beam on the gold foil). The probe beam was focused by a f/10 parabola to a spot size of $50 \mu \mathrm{m}$ onto a $12.5 \mu \mathrm{m}$ thick Ti foil to generate short pulse line radiation at $4.5 \mathrm{keV}$. Both the pump and probe lasers were incident normal to the foil and the $x$ rays intercepted the gold foil at the Bragg angle $\left(\theta_{B}=35.9^{\circ}\right)$. A schematic of the experimental setup is shown in Fig. 1.

The $\mathrm{x}$-ray line radiation was scattered coherently from the gold nanofoil and imaged onto a $2 \mathrm{~cm} \times 2 \mathrm{~cm}$ charged coupled device (CCD) array with $13.5 \mu \mathrm{m}$ pixel size, placed at a distance of $10 \mathrm{~cm}$ from the sample. The diffraction angle, and hence the position of the line on the CCD chip, is related to the interplanar distance in gold through Bragg's law. Thus, any movement of the atoms such as thermal expansion can be seen through translation of the diffraction line.

For each shot a Gaussian profile was fitted to the middle of the diffraction line and the center of the Gaussian used to calculate the diffraction angle. Multiple shots were taken for each pump-probe delay and the error bars were estimated using one standard deviation from the average. For delays between -5 and 40 ps each condition was repeated 12 times, for all other delays an average of 6 shots were used. The experimentally obtained change in diffraction angle including standard deviation in the error bars is shown in Fig. 2.

\section{MODELING THE LATTICE RESPONSE}

A phenomenological model describing the energy flow between the electron and phonon subsystems and thus the temperature relaxation is given by [23]

$$
\begin{aligned}
C_{e} \frac{\partial T_{e}}{\partial t} & =\nabla\left(K_{e} \nabla T_{e}\right)-g\left(T_{e}-T_{i}\right)+S_{e}(t), \\
C_{i} \frac{\partial T_{i}}{\partial t} & =\nabla\left(K_{i} \nabla T_{i}\right)+g\left(T_{e}-T_{i}\right),
\end{aligned}
$$

where $C_{e}\left(C_{i}\right)$ is the electron (phonon) specific heat capacity, $K_{e}\left(K_{i}\right)$ is the electron (phonon) thermal conductivity, and
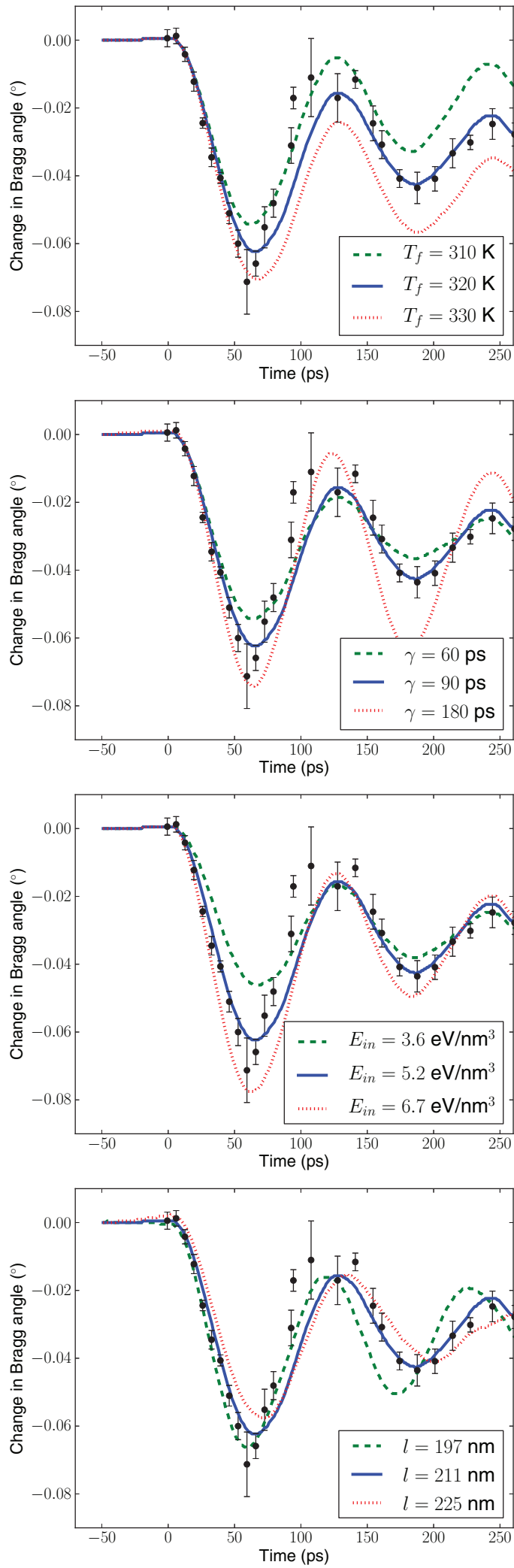

FIG. 2. (Color online) Change in diffraction angle from the gold nanofoil compared to results from MD simulations. The effect of changing the (from top to bottom) final temperature of the lattice; the damping coefficient; the energy absorbed by the lattice per nanometre cube and the thickness of the gold. The solid line represents the best fit, while the dashed and dotted lines are under- and overestimates, respectively. 
$S_{e}(t)$ is the source term due to the heating by the laser incident on the sample. This model is usually referred to as the two-temperature model (TTM). The transfer rate is set by the coupling constant $g$. This method allows the complexity of the physical system to be contained within a single parameter. The coupling constant is a quantity which is difficult to estimate from first principles as it depends on the interaction dynamics of the electron and phonon subsystems. We expect its value to be constant with temperature under the small range of conditions found in our experiment [11].

Given the large laser spot size compared to the thickness of the gold foil, we can treat the heating of the sample as being one dimensional. Furthermore the source term in Eq. (1a) does not depend on a spatial coordinate and, thus, does not take into account the finite penetration depth of the laser. This is due to the ballistic nature of the hot electrons which causes the target to equilibrate spatially across its depth within a picosecond after laser irradiation [24-26]. As the energy is transferred from the hot electron population to the ions forming the lattice, the crystal expands and the lattice parameter increases. From Bragg's law, the position of the diffraction line can be related to the expansion of the sample. If the equilibration rate is quick (less than a few tens of ps) the gold foil will rapidly expand and oscillate around some new mean lattice constant. If the rate is slower the sample will slowly expand until it reaches the new lattice constant. In our experiment we expect the former scenario to apply (see for example Ref. [27]).

Applicability of the TTM requires that both the electron and phonon subsystems are in local equilibrium, that is the electron-electron scattering maintains a Fermi-Dirac distribution with a characteristic temperature $T_{e}$ and phononphonon scattering maintains a Bose-Einstein distribution with a characteristic temperature $T_{i}$ [28]. The electron-electron thermalization time can be estimated from the relation $\tau_{e} \approx$ $\hbar E_{F} / 4\langle E\rangle^{2}$ where $E_{F}$ is the Fermi energy and $\langle E\rangle$ the mean electron energy [29]. For the gold nanofoil used here, $E_{F}=$ $5.53 \mathrm{eV}$. For an average electron energy of $\langle E\rangle=0.086 \mathrm{eV}$, which corresponds to an electron temperature of $1000 \mathrm{~K}$, the electron-electron thermalization time is of the order of $0.25 \mathrm{ps}$. Given the laser fluence in this experiment we expect electron temperatures of $1000 \mathrm{~K}$ to be readily accessible [29]. The electron-electron energy equilibration, spatial equilibration, and laser pulse duration are all much less than the timescale of interest in this work, and hence it is reasonable to approximate the source term in the TTM to be instantaneous, i.e., $S_{e}(t) \approx$ $S_{e} \delta(t)$. Indeed, in previous experiments on thin gold films of a similar thickness to those used here the electron distribution was found to be Fermi-Dirac-like after 0.8 ps [29]. Therefore, a full nonequilibrium treatment of the electron subsystem, which has been shown to modify the energy transfer to the lattice [8], is not necessary here and we can rely on temperature estimates.

In contrast, the phonon-phonon thermalization time for gold is much longer, and it has been estimated theoretically to be of the order of tens of picoseconds. Hence the phonon system is expected to be out of local equilibrium on the timescales probed here. However, since the change in phonon temperature found in our experiment remains small (i.e., a $10 \%$ increase from 300 to $\sim 330 \mathrm{~K}$ ), we can still describe the phonon gas in terms of a Bose-Einstein distribution at $300 \mathrm{~K}$ plus a small number of out-of-equilibrium phonons [29], and assume that the additional excitation of the phonon system results in negligible changes of the energy transfer during equilibration. The evolution of the electron and ion temperatures in the sample is dominated by the electron-phonon coupling, and this occurs on timescales of the order of a few picoseconds.

In order to understand the details of the equilibration process, we have modeled the gold foil in a classical molecular dynamics simulation. Molecular dynamics is a powerful tool that has had considerable success in describing the lattice dynamics in numerous laser-solid target interactions [30]. Here, we vary the rate at which energy is transferred to the ions to match the experimental results. This is made possible by the one dimensional nature of the experiment. Classical molecular dynamics simulations are performed in the LAMMPS package [31] with a microcanonical ensemble. The simulation used 114916 atoms in a $3.98 \mathrm{~nm} \times 2.30 \mathrm{~nm} \times 218 \mathrm{~nm}$ box which is equal in size to $3 \times 3 \times 300$ gold crystal unit cells. The small cross section preserves the one-dimensional nature of the problem. The simulation box was split into $7 \mathrm{~nm}$ of vacuum and $211 \mathrm{~nm}$ of gold with the crystal lattice orientated along the (111) plane.

Each simulation was run with a 1 fs time step and the total number of time steps in each simulation was 300000 . The simulations were run for 50000 time steps prior to the application of the laser heating to ensure that the crystal was initially at rest. A viscous damping factor, $\gamma$, is applied to the gold lattice in order to account for the energy loss to the mica substrate. This factor allows the adjustment of the speed with which the gold loses energy.

The gold atoms are modeled using an embedded atom model potential [32]. This potential accurately predicts the lattice constant and bulk modulus of gold to within $2 \%$ and we therefore expect to accurately capture the acoustic dynamics. The molecular dynamics simulation was run by accounting for the energy relaxation between electrons and ions via the TTM. We utilize an exponential function as an approximate solution to Eqs. (1a) and (1b) for a given initial electron temperature. That is, energy is deposited into the ion subsystem by exponentially decreasing amounts with a time constant equal to $\tau=C_{e} / g$, where $g$ is the electron-phonon equilibration constant [35]. We take the electron heat capacity to have a value of $C_{e}=67.6 T_{e} \mathrm{~J} \mathrm{~m}^{-3} \mathrm{~K}^{-2}$ [11]. This solution to the TTM assumes that the electron and lattice thermal conductivites are low enough as to not transport heat laterally in the target over the timescales of the experiment. For gold in 250 ps the characteristic length over which heat is conducted is $(K t / C)^{\frac{1}{2}}=200 \mathrm{~nm}$, considerably less than the $3 \mathrm{~mm}$ heated spot. Finally, the predicted diffraction line is obtained from the Fourier transform of the atom positions at each time step. The peak position in reciprocal space is related to the diffraction angle by $k=4 \pi\left(E_{0} / h c\right) \sin (\theta / 2)$ where $E_{0}$ is the energy of diffracted x rays and $k$ the scattering wavenumber.

As electrons are heated by the laser, a sound wave is launched into the bulk of the sample. The oscillations shown in the diffraction peak position for delay times $\Delta t \gtrsim 40 \mathrm{ps}$ (see Fig. 2) are related to reverberation of the sound wave between the sides of the sample. This process occurs on timescales longer than the energy equilibration between electron and ions $(\tau \lesssim 10 \mathrm{ps}$ ), and it can thus be used in order to infer 


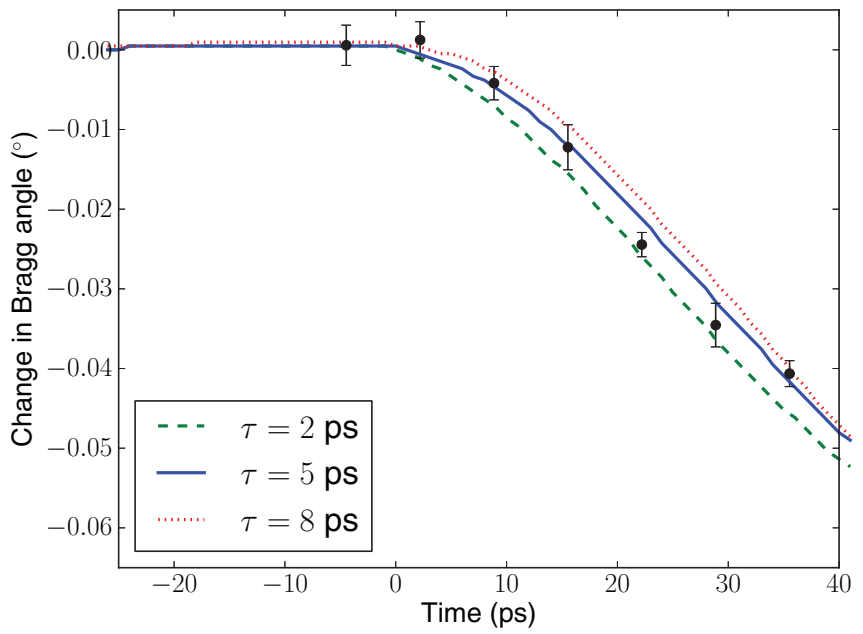

FIG. 3. (Color online) The variation of $\tau$ in the simulation. The solid line represents the best fit ( $\tau=5 \mathrm{ps}$ ) while the dashed and dotted lines are under and over-estimates ( $\tau=2 \mathrm{ps}, 8 \mathrm{ps})$ respectively. We have used $\gamma=90 \mathrm{ps}, T_{f}=320 \mathrm{~K}, E_{\text {in }}=5.2 \mathrm{eV} / \mathrm{nm}^{3} l=211 \mathrm{~nm}$ in all cases.

the long-term mechanical response of the gold due to the short-time perturbation caused by the laser heating.

There are four free parameters available in the numerical simulation which must be varied in order to fit the data for $\Delta t \gtrsim 40 \mathrm{ps}$. These are the thickness of the gold, $l$, which is known with $\pm 20 \%$ accuracy, the total energy absorbed into the gold target per nanometre cube, $E_{\text {in }}$, the final temperature of the lattice, $T_{f}$, and the damping coefficient, $\gamma$. Figure 2 shows the change in predicted behavior associated with varying each of these parameters. The effect of the choice of a different final temperature is to change the equilibrium lattice spacing at late time, while the damping coefficient controls the time over which the lattice attains this new configuration. The energy absorbed determines primarily the depth of the first trough as well as the amount of ringing seen about the equilibrium lattice spacing. Lastly, the gold thickness alters the period of the oscillations. Our best fit values for these parameters are $\gamma=90 \mathrm{ps}, T_{f}=320 \mathrm{~K}, E_{\text {in }}=5.2 \mathrm{eV} / \mathrm{nm}^{3}$, and $l=211 \mathrm{~nm}$.

Once the long-time behavior has been determined, the simulation is used to fit the short-time behavior ( $\Delta t<40 \mathrm{ps}$ ), which is strongly determined by the electron-phonon coupling parameter $(g)$ in the TTM. In the simulations we have varied the energy relaxation time, $\tau$, to best fit the data, as shown in Fig. 3. We find a value of $\tau=5 \pm 3$ ps. This value agrees with reported data in a similar experiment [9] where it was inferred by observing the shift in position of the peaks due to
TABLE I. Comparison of electron-phonon coupling times in gold reported in the literature together with the result from this work. The time constant $\tau$ is given in units of ps and the coupling constant $g$ is given in units of $\mathrm{W} \mathrm{m}^{-3} \mathrm{~K}^{-1}$. Values marked with a $\dagger$ have been inferred from $\tau=C_{e}\left(T_{e}\right) / g$.

\begin{tabular}{llcc}
\hline \hline Expt. method & \multicolumn{1}{c}{$\tau$} & $\mathrm{g} / 10^{16}$ & Reference \\
\hline Bragg peak shift (MD) & $5.0 \pm 3$ & $2.0 \pm 1.2$ & this work \\
Bragg peak shift (acoustic) & $5.0 \pm 0.3$ & $1.6 \pm 0.1 \dagger$ & {$[9]$} \\
Bragg peak intensity & $4.7 \pm 0.6$ & $1.8 \pm 0.2 \dagger$ & {$[33]$} \\
Surface expansion & $1.5 \pm 1.0 \dagger$ & $1.6 \pm 0.6$ & {$[34]$} \\
Surface reflectivity & $4.0 \pm 1.0 \dagger$ & $2.2 \pm 0.3$ & {$[35]$} \\
A $b$ initio calculation & & 2.5 & {$[11]$} \\
\hline \hline
\end{tabular}

acoustic reverberation in the sample. However, our analysis indicates that other factors (in particular the energy loss to the mica substrate) may also determine the position of those peaks which are not directly related to the energy relaxation time (see Fig. 2). We therefore believe that our results thus give a more reliable estimate of the equilibration time than previous work.

\section{CONCLUSIONS}

The determined value for $\tau$ leads to an electron-phonon equilibration constant of $g=2 \pm 1.2 \times 10^{16} \mathrm{~W} \mathrm{~m}^{-3} \mathrm{~K}^{-1}$, a value which is in close agreement with previous studies. Table I contains a comparison of electron-phonon coupling constants based on a variety of experimental and theoretical techniques. This result agrees well with previous values, confirming that the energy relaxation in gold is a fast process in the bulk as well as on the surface of the sample. The fact that different experimental methods yield very similar values for the electron-phonon coupling constant strongly raises the confidence in this result at low excitations. This finding, in combination with the discrepancies for higher energy input both for solids and fluids, further points towards changes in the relaxation behavior in strongly driven systems. This work highlights the possibility to study energy relaxation processes in bulk samples through large-scale MD simulations and could be particularly successful at describing the complex ion-ion correlations [36,37] which make experiments with higher excitation densities difficult to describe [20,21].

\section{ACKNOWLEDGMENTS}

This work was supported in parts by the Science and Technology Facilities Councils, EPSRC (Grant No. EP/G007187/1), and by AWE plc.
[1] J. Bardeen, L. N. Cooper, and J. R. Schrieffer, Phys. Rev. 108, 1175 (1957).

[2] N. W. Ashcroft and N. D. Mermin, Solid State Physics (Saunders, Philadelphia, 1976).

[3] M. C. Klein, F. Hache, D. Ricard, and C. Flytzanis, Phys. Rev. B 42, 11123 (1990).

[4] S.-S. Wellershoff, J. Hohlfeld, J. Güdde, and E. Matthias, Appl. Phys. A 69, S99 (1999).
[5] D. Bäuerle, Laser Processing and Chemistry (Springer, Berlin, 2011).

[6] S. Atzeni and J. Meyer-ter-Vehn, The Physics of Inertial Fusion (Oxford University Press. Oxford, 2009).

[7] D. O. Gericke, S. Kosse, M. Schlanges, and M. Bonitz, Phys. Rev. B 59, 10639 (1999).

[8] B. Y. Mueller and B. Rethfeld, Phys. Rev. B 87, 035139 (2013). 
[9] M. Nicoul, U. Shymanovich, A. Tarasevitch, D. von der Linde, and K. Sokolowski-Tinten, Appl. Phys. Lett. 98, 191902 (2011).

[10] T. G. White, J. Vorberger, C. R. D. Brown, B. J. B. Crowley, P. Davis, S. H. Glenzer, J. W. O. Harris, D. C. Hochhaus, S. Le Pape, T. Ma, C. D. Murphy, P. Neumayer, L. K. Pattison, S. Richardson, D. O. Gericke, and G. Gregori, Sci. Rep. 2, 889 (2012).

[11] Z. Lin, L. V. Zhigilei, and V. Celli, Phys. Rev. B 77, 075133 (2008).

[12] B. L. Altshuler, A. G. Aronov, and P. A. Lee, Phys. Rev. Lett. 44, 1288 (1980).

[13] P. M. Leguay, A. Levy, B. Chimier, F. Deneuville, D. Descamps, C. Fourment, C. Goyon, S. Hulin, S. Petit, O. Peyrusse, J. J. Santos, P. Combis, B. Holst, V. Recoules, P. Renaudin, L. Videau, and F. Dorchies, Phys. Rev. Lett. 111, 245004 (2013)

[14] Z. Chen, B. Holst, S. E. Kirkwood, V. Sametoglu, M. Reid, Y. Y. Tsui, V. Recoules, and A. Ng, Phys. Rev. Lett. 110, 135001 (2013).

[15] P. Celliers, A. Ng, G. Xu, and A. Forsman, Phys. Rev. Lett. 68, 2305 (1992).

[16] A. Ng, P. Celliers, G. Xu, and A. Forsman, Phys. Rev. E 52, 4299 (1995).

[17] D. Riley, N. C. Woolsey, D. McSherry, I. Weaver, A. Djaoui, and E. Nardi, Phys. Rev. Lett. 84, 1704 (2000).

[18] T. G. White, N. J. Hartley, B. Borm, B. J. B. Crowley, J. W. O. Harris, D. C. Hochhaus, T. Kaempfer, K. Li, P. Neumayer, L. K. Pattison, F. Pfeifer, S. Richardson, A. P. L. Robinson, I. Uschmann, and G. Gregori, Phys. Rev. Lett. 112, 145005 (2014).

[19] M. W. C. Dharma-wardana and F. Perrot, Phys. Rev. E 58, 3705 (1998).

[20] G. Gregori and D. O. Gericke, Europhys. Lett. 83, 15002 (2008).

[21] J. Vorberger, D. O. Gericke, Th. Bornath, and M. Schlanges, Phys. Rev. E 81, 046404 (2010).
[22] The goil foil is commercially available from PHASIS (Switzerland) and has been epitaxial grown onto a mica muscovite substrate.

[23] S. I. Anisimov, B. L. Kapeliovich, and T. L. Perel'man, Zh. Eksp. Teor. Fiz. 66, 776 (1974) [Sov. Phys._JETP 39, 375 (1975)].

[24] S. D. Brorson, J. G. Fujimoto, and E. P. Ippen, Phys. Rev. Lett. 59, 1962 (1987).

[25] T. Juhasz, H. E. Elsayed-Ali, G. O. Smith, C. Suarez, and W. E. Bron, Phys. Rev. B 48, 15488 (1993).

[26] C. Suarez, W. E. Bron, and T. Juhasz, Phys. Rev. Lett. 75, 4536 (1995).

[27] C. Rose-Petruck, R. Jimenez, T. Guo, A. Cavalleri, C. W. Siders, F. Rksi, J. A. Squier, B. C. Walker, K. R. Wilson, and C. P. J. Barty, Nature (London) 398, 310 (1999).

[28] M. Agranat, S. Anisimov, and B. Makshantsev, Appl. Phys. B 47, 209 (1988).

[29] H. E. Elsayed-Ali and T. Juhasz, Phys. Rev. B 47, 13599 (1993).

[30] K. Kadau, T. C. Germann, P. S. Lomdahl, and B. L. Holian, Science 296, 1681 (2002).

[31] S. Plimpton, J. Comput. Phys. 117, 1 (1995).

[32] H. W. Sheng, M. J. Kramer, A. Cadien, T. Fujita, and M. W. Chen, Phys. Rev. B 83, 134118 (2011).

[33] M. Ligges, I. Rajkovic, P. Zhou, O. Posth, C. Hassel, G. Dumpich and D. von der Linde, Appl. Phys. Lett. 94, 101910 (2009).

[34] O. B. Wright, Phys. Rev. B 49, 9985 (1994).

[35] J. Hohlfeld, S.-S. Wellershoff, J. Güdde, U. Conrad, V. Jähnke, and E. Matthias, Chem. Phys. 251, 237 (2000).

[36] T. Ma, T. Doppner, R. W. Falcone, L. Fletcher, C. Fortmann, D. O. Gericke, O. L. Landen, H. J. Lee, A. Pak, J. Vorberger, K. Wunsch, and S. H. Glenzer, Phy. Rev. Lett. 110, 065001 (2013).

[37] D. Kraus, J. Vorberger, D. O. Gericke, V. Bagnoud, A. Blazevic, W. Cayzac, A. Frank, G. Gregori, A. Ortner, A. Otten, F. Roth, G. Schaumann, D. Schumacher, K. Siegenthaler, F. Wagner, K. Wunsch, and M. Roth, Phys. Rev. Lett. 111, 255501 (2013). 